\author{
Marquette University \\ e-Publications@Marquette
}

Biomedical Engineering Faculty Research and

Publications

Biomedical Engineering, Department of

$5-2003$

\title{
Reactive Oxygen Species Modulate Coronary Wall Shear Stress and Endothelial Function During Hyperglycemia
}

\author{
Eric R. Gross \\ Medical College of Wisconsin \\ John F. LaDisa \\ Marquette University, john.ladisa@marquette.edu \\ Dorothee Weihrauch \\ Medical College of Wisconsin \\ Lars E. Olson \\ Marquette University, lars.olson@marquette.edu \\ Tobias T. Kress \\ Medical College of Wisconsin
}

See next page for additional authors

Follow this and additional works at: https://epublications.marquette.edu/bioengin_fac

Part of the Biomedical Engineering and Bioengineering Commons

\section{Recommended Citation}

Gross, Eric R.; LaDisa, John F.; Weihrauch, Dorothee; Olson, Lars E.; Kress, Tobias T.; Hettrick, Douglas Anthony; Pagel, Paul S.; Warltier, David C.; and Kersten, Judy R., "Reactive Oxygen Species Modulate Coronary Wall Shear Stress and Endothelial Function During Hyperglycemia" (2003). Biomedical Engineering Faculty Research and Publications. 219.

https://epublications.marquette.edu/bioengin_fac/219 


\section{Authors}

Eric R. Gross, John F. LaDisa, Dorothee Weihrauch, Lars E. Olson, Tobias T. Kress, Douglas Anthony Hettrick, Paul S. Pagel, David C. Warltier, and Judy R. Kersten 
Marquette University

e-Publications@Marquette

\section{Biomedical Engineering Faculty Research and Publications/College of} Engineering

This paper is NOT THE PUBLISHED VERSION; but the author's final, peer-reviewed manuscript. The published version may be accessed by following the link in the citation below.

American Journal of Physiology : Heart and Circulatory Physiology, Vol. 284, No. 5 (May 2003): H1552$\mathrm{H1559}$. DOI. This article is (C) American Physiological Society and permission has been granted for this version to appear in e-Publications@Marquette. American Physiological Society does not grant permission for this article to be further copied/distributed or hosted elsewhere without the express permission from American Physiological Society.

\section{Reactive oxygen species modulate coronary wall shear stress and endothelial function during hyperglycemia}

\section{Eric R. Gross}

Departments of Anesthesiology, Pharmacology and Toxicology, Medical College of Wisconsin Clement J. Zablocki Department of Veterans Affairs Medical Center, Milwaukee Department of Biomedical Engineering, Marquette University, Milwaukee, Wisconsin John F. LaDisa Jr.

Departments of Anesthesiology, Department of Biomedical Engineering, Marquette University, Milwaukee, Wisconsin

Dorothee Weihrauch

Departments of Anesthesiology, Department of Biomedical Engineering, Marquette University, Milwaukee, Wisconsin

Lars E. Olson

Department of Biomedical Engineering, Marquette University, Milwaukee, Wisconsin 


\section{Tobias T. Kress}

Departments of Anesthesiology, Department of Biomedical Engineering, Marquette University, Milwaukee, Wisconsin

\section{Douglas A. Hettrick}

Departments of Anesthesiology, Department of Biomedical Engineering, Marquette University, Milwaukee, Wisconsin

\section{Paul S. Pagel}

Departments of Anesthesiology, Department of Biomedical Engineering, Marquette University, Milwaukee, Wisconsin

\section{David C. Warltier}

Departments of Anesthesiology, Pharmacology and Toxicology, Medical College of Wisconsin and Clement J. Zablocki Department of Veterans Affairs Medical Center, Milwaukee Department of Biomedical Engineering, Marquette University, Milwaukee, Wisconsin

\section{Judy R. Kersten}

Departments of Anesthesiology, Pharmacology and Toxicology, Medical College of Wisconsin and Clement J. Zablocki Department of Veterans Affairs Medical Center, Milwaukee, Wisconsin

\section{Abstract}

Hyperglycemia is associated with generation of reactive oxygen species (ROS), and this action may contribute to accelerated atherogenesis. We tested the hypothesis that hyperglycemia produces alterations in left anterior descending coronary artery (LAD) wall shear stress concomitant with endothelial dysfunction and ROS production in dogs $(n=12)$ instrumented for measurement of LAD blood flow, velocity, and diameter. Dogs were randomly assigned to receive vehicle ( $0.9 \%$ saline) or the superoxide dismutase mimetic 4 - hydroxy-2,2,6,6tetramethylpiperidine-1-oxyl (tempol) and were administered intravenous infusions of d-glucose to achieve target blood glucose concentrations of 350 and $600 \mathrm{mg} / \mathrm{dl}$ (moderate and severe hyperglycemia, respectively). Endothelial function and ROS generation were assessed by coronary blood flow responses to acetylcholine (10, 30 , and $100 \mathrm{ng} / \mathrm{kg}$ ) and dihydroethidium fluorescence of myocardial biopsies, respectively. Indexes of wall shear stress were calculated with conventional fluid dynamics theory. Hyperglycemia produced dose-related endothelial dysfunction, increases in ROS production, and reductions in oscillatory shear stress that were normalized by tempol. The results suggest a direct association between hyperglycemia-induced ROS production, endothelial dysfunction, and decreases in oscillatory shear stress in vivo.

reactive oxygen species (ROS) have been implicated in the pathogenesis of cardiovascular disease during diabetes mellitus and hyperglycemia. Several potential mechanisms have been identified that may account for increases in ROS generation during hyperglycemia including glucose autooxidation (2), mitochondrial superoxide anion overproduction by the electron transport chain (32), depletion of tetrahydrobiopterin (34), and synthesis of advanced glycation end-products (36). Hyperglycemia inhibits endothelium-dependent coronary vasodilation through oxidant-sensitive mechanisms $(20,21,38)$. A disproportionate increase in superoxide anion compared with nitric oxide (NO) concentrations occurs during hyperglycemia that may account for endothelial dysfunction by altering the balance of oxidant stress $(2,5,6)$, and this effect is attenuated by free radical scavengers that indirectly increase the availability of NO $(2,29,35,38)$.

Hyperglycemia has also been shown to adversely alter vascular fluid dynamics. An inverse relation between fasting blood glucose concentrations and wall shear stress has been observed in patients with diabetes compared with age-matched controls $(15,16)$, and endothelium-derived relaxing factors including NO are 
modulated by wall shear stress in a frequency- and amplitude-dependent manner $(11,12)$. Regions of the arterial vasculature that are chronically exposed to low-magnitude and oscillatory wall shear stress are known to be more susceptible to the development of atherogenesis $(9,23)$. Collectively, these studies suggest that hyperglycemia-induced ROS production may alter endothelial function and account for reductions in indexes of wall shear stress. To date, no studies have examined the direct effects of hyperglycemia on coronary wall shear stress, ROS production, and endothelial function simultaneously in vivo. We tested the hypothesis that hyperglycemia-induced ROS production is linked to coronary endothelial dysfunction and decreases in wall shear stress in dogs.

\section{MATERIALS AND METHODS}

All experimental procedures and protocols used in this investigation were reviewed and approved by the Institutional Animal Care and Use Committee of the Medical College of Wisconsin. All conformed to the Guiding Principles in the Care and Use of Animals of the American Physiological Society and the Guide for the Care and Use of Laboratory Animals of the National Institutes of Health (7th ed., Washington, DC: National Academy Press, 1996).

\section{Surgical preparation.}

Implantation of instruments was described previously (24). Adult mongrel dogs of either sex and weighing between 18 and $24 \mathrm{~kg}$ were fasted overnight and anesthetized with barbital $(200 \mathrm{mg} / \mathrm{kg}$ ) and pentobarbital sodium $(15 \mathrm{mg} / \mathrm{kg})$. After endotracheal intubation, dogs were ventilated using positive pressure with an airoxygen mixture $\left(\mathrm{Fi}_{\mathrm{O} 2}=0.28-0.30\right)$. Arterial blood gas tensions and acid-base status were maintained within physiological limits by adjusting tidal volume and respiratory rate. Fluid-filled catheters were inserted into the right femoral vein and artery for fluid administration and arterial blood gas sampling, respectively. A thoracotomy was performed in the fifth intercostal space, the lungs were gently retracted, and the pericardium was incised. A dual micromanometer-tipped catheter was inserted into the left ventricle (LV) and ascending aorta for measurement of LV and arterial pressures, respectively, and the maximum rate of increase of LV pressure $\left(\mathrm{dP} / \mathrm{d} t_{\max }\right)$. A 1.5- to 2-cm segment proximal to the first diagonal branch of the left anterior descending coronary artery (LAD) was isolated, and 2.5- $\mathrm{mm}$ ultrasonic segment length (Triton, San Diego, CA) and transit time blood flow (Transonic Systems, Ithaca, New York) transducers were placed around the vessel for measurement of external diameter and regional blood flow (Fig.1A). A precalibrated 20-MHz pulsed Doppler flow probe was placed around the LAD just distal to the first diagonal branch to measure local blood flow velocity at the myocardial and pericardial luminal surfaces (Fig. 1B). A fluid-filled catheter was inserted into the left atrium through the atrial appendage for administration of acetylcholine. Fluid deficits were replaced before experimentation with $500 \mathrm{ml}$ of $0.9 \%$ saline, which was continued at $3 \mathrm{ml} \cdot \mathrm{kg}^{-1} \cdot \mathrm{h}^{-1}$ for the duration of each experiment. Hemodynamic data were continuously monitored on a polygraph and simultaneously digitized (364 $\mathrm{Hz}$ ) with a computer interfaced with an analog-to-digital converter. 


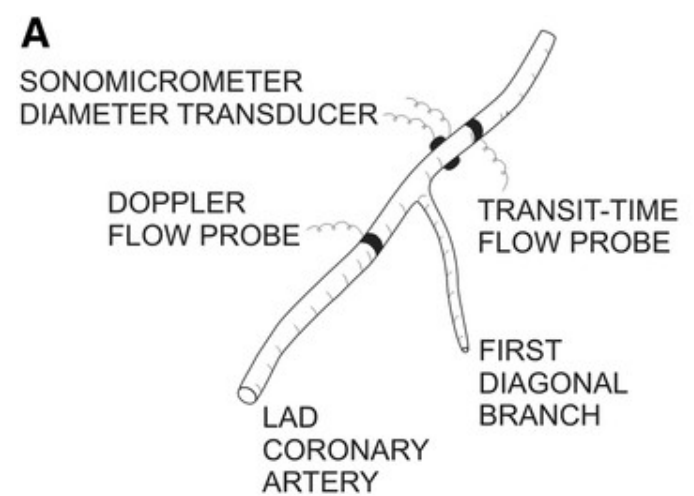

\section{B}

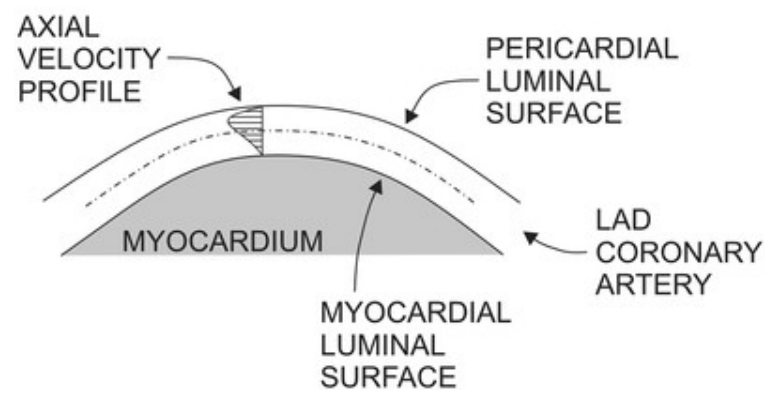

Fig. 1.A: schematic diagram of the instrumentation used to measure left anterior descending coronary artery (LAD) blood flow velocity and LAD external diameter. $B$ : location of the myocardial and pericardial luminal surfaces.

\section{Experimental protocol.}

Baseline systemic and coronary hemodynamics were recorded 90 min after instrumentation was completed. Dogs were randomly assigned to receive $0.9 \%$ saline (vehicle; $n=6$ ) or the superoxide dismutase mimetic 4hydroxy-2,2,6,6-tetramethylpiperidine-1-oxyl (tempol; $100 \mathrm{mg} / \mathrm{kg}$ in $0.9 \%$ saline over $15 \mathrm{~min}$; Sigma, St. Louis, $\mathrm{MO} ; n=6)$. Hemodynamics were acquired under steady-state conditions after vehicle or tempol pretreatment and during acetylcholine infusions (10,30, and $100 \mathrm{ng} / \mathrm{kg}$ ) (27). Tissue samples were removed with a biopsy needle (Bard, Billerica, MA) during each intervention from the left circumflex coronary artery perfusion territory for measurement of ROS. Blood glucose concentrations and arterial blood gas tensions were determined during each intervention. After the administration of vehicle or tempol and acetylcholine, blood glucose concentrations were sequentially increased to 350 and $600 \mathrm{mg} / \mathrm{dl}$ (moderate and severe hyperglycemia, respectively) with an intravenous infusion of $d$-glucose. Target concentrations of blood glucose were selected on the basis of a previous investigation demonstrating the ability of hyperglycemia to cause dose-dependent reductions in coronary collateral blood flow in dogs (19). Each target blood glucose concentration was maintained for 90 min, and systemic and coronary hemodynamics before and during administration of acetylcholine were recorded. Myocardial biopsies were also obtained during each intervention in the presence or absence of hyperglycemia.

\section{Detection of ROS.}

Production of ROS was quantified during each intervention from myocardial biopsy samples with the fluorescent dye dihydroethidium. Dihydroethidium is oxidized by intracellular ROS to produce fluorescent ethidium that is subsequently intercalated in DNA, further amplifying its fluorescence (4). In cell-free assays, the addition of hydrogen peroxide to dihydroethidium does not increase ethidium fluorescence (28). Thus an increase in dihydroethidium oxidation and the subsequent increase in fluorescence are highly suggestive of superoxide generation. Myocardial biopsy samples were incubated in PBS at $37^{\circ} \mathrm{C}$ overnight, washed with PBS, and incubated in $5 \mu \mathrm{M}$ dihydroethidium for $30 \mathrm{~min}$. Images were obtained from each intervention with a laser 
fluorescence imaging system (Odyssey Laser Scanning Confocal Microscopic Imaging System; Noran Instruments, Madison, WI) mounted on a microscope (Optiphot; Nikon, Tokyo, Japan) and stored for off-line analysis on a computer workstation equipped with image-analysis software (MetaMorph; Universal Imaging, Downingtown, PA). Excitation was produced with a krypton-argon laser at a wavelength of $488 \mathrm{~nm}$ and emitted fluorescence was measured at $610 \mathrm{~nm}$. The pixel intensity of each myocyte nucleus was determined after compensating for background fluorescence by subtraction of an area without cells or minimal cytosolic fluorescence. For each experiment, 7 images were obtained at each intervention and the intensity of 10 myocardial nuclei were analyzed in each image.

\section{Determination of endothelial function.}

Endothelial function was quantified by the coronary vasodilator response to acetylcholine (27). Acetylcholine infusions were administered into the left atrial appendage during each intervention at a rate of $0.5 \mu \mathrm{l} / \mathrm{s}$ for $4 \mathrm{~s}$. The rate and duration of acetylcholine administration were selected to achieve increases in coronary blood flow similar to those described previously (27). Hemodynamics were allowed to return to steady-state conditions before subsequent doses of acetylcholine were administered.

\section{Ensemble averaging of hemodynamics.}

LV and aortic blood pressure, LAD blood flow and velocity, and LAD external diameter waveforms were digitally recorded for each intervention. A representative time series of one cardiac cycle for each waveform was generated with a custom-designed MATLAB (MathWorks, Natick, MA) program that identified peak LV pressure to temporally align the variables in each digitized waveform, segment each variable time series, and ensembleaverage the segments.

\section{Determination of internal LAD diameter.}

The LAD coronary artery was excised, and the length $(L)$ of the vascular segment was determined in situ at the completion of each experiment. The internal LAD radius $\left(r_{\mathrm{i}}\right)$ was determined with the equation $r_{\mathrm{i}}=$ $(r 2002-V / L \pi)^{1 / 2}$, wherer20o2 is the external LAD radius and $V$ is the ratio of the excised vessel weight to density (assumed as $1.06 \mathrm{~g} / \mathrm{ml}$ ) (31).

\section{Determination of local coronary blood flow velocity.}

Local blood flow velocity at the pericardial and myocardial luminal surfaces (Fig. 1B) of the LAD segment was determined using pulsed Doppler techniques $(1,24,26)$. Briefly, a piezoelectric crystal with a stationary predetermined insonation angle that is fixed within a Doppler probe is deformed, emitting an ultrasonic pulse that propagates through the vessel wall to a specified depth. The pulse is then reflected back to the crystal and interpreted as a frequency shift. To ensure repeatability between interventions and experiments, Doppler pulse penetration depth had been previously correlated to values on a digital voltmeter. Digitized sample volumes $\left(\sim 0.23 \mathrm{~mm}^{3}\right)$ were obtained at 12 axial depths across the vessel and converted to blood flow velocity by determination of insonation angle as previously described (1). Velocity waveforms at each axial depth were ensemble-averaged and spatially aligned to reconstruct velocity profiles for each intervention. Least-squares interpolation was then performed to acquire near-wall velocity assuming no slip at the vessel wall.

\section{Determination of blood viscosity.}

Blood samples ( $3 \mathrm{ml}$ each) were withdrawn from the femoral artery catheter during each intervention for measurement of blood viscosity. Samples were placed in EDTA-containing vials and agitated continuously to maintain uniform cellular composition. Blood viscosity was measured in duplicate at $37^{\circ} \mathrm{C}$ for five different shear rates $\left(375,187.5,75,56.25\right.$, and $30 \mathrm{~s}^{-1}$ ) with a cone and plate viscometer (DVII+; Brookfield Engineering Labs, Stoughton, MA). Blood viscosity was then plotted against shear rate, and equations describing a three-constant 
exponential decay relationship of the curve were obtained for each intervention by using a MarquardtLevenberg algorithm with commercially available software (Sigma Plot 2000; SPSS, Chicago, IL).

\section{Determination of global and local indexes of shear stress.}

Shear rate was determined by two methods. Global shear rate $(\gamma)$ within the LAD vascular segment bounded by the surgical instrumentation was estimated from Poiseuille's law using the equation $\gamma=4 Q^{\circ} \pi r^{\prime} \mathrm{ii}^{\prime}$, where $Q^{\prime}$ is the rate of coronary blood flow. Local wall shear rate was calculated from blood velocity measurements at the LAD coronary artery walls nearest to the pericardial and myocardial luminal surfaces (Fig. $1 B$ ) by a finite difference method using the differential equation $\gamma_{u}=-\partial u / \partial r$, where $\partial u / \partial r$ is the partial derivative of the velocity magnitude with respect to the radial vessel position $(r)(13)$. Global $(\tau)$ and local $\left(\tau_{u}\right)$ shear stress for each time point in the cardiac cycle were calculated as the product of the measured in vivo viscosity and the respective global or local shear rate. Oscillatory shear stress $\left(\tau_{o s}\right)$ was determined as the magnitude of the shear stress waveform (Fig. 2 ). The rate of oscillatory shear stress ( $\tau_{\text {os }}^{\circ}$ ) was also calculated as the product of $\tau_{\text {os }}$ and heart rate.

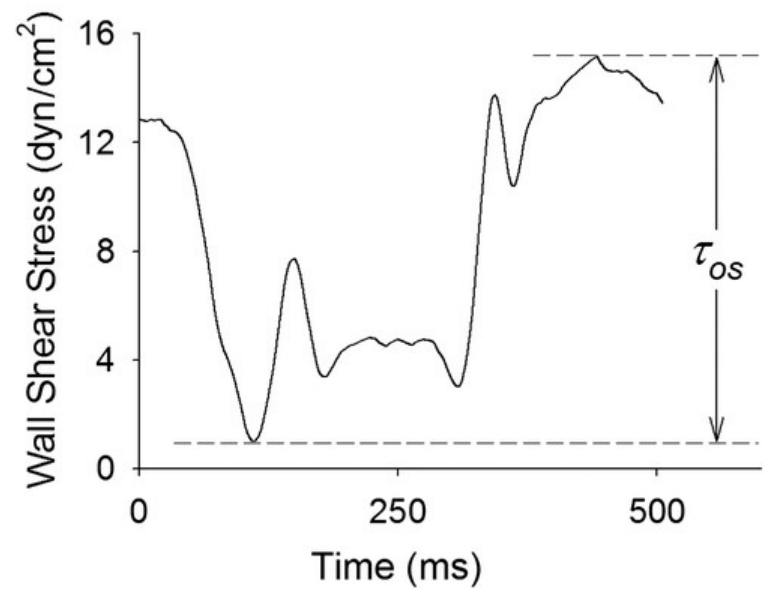

Fig. 2.Ensemble-averaged wall shear stress waveform obtained from a typical experiment during baseline conditions. Oscillatory wall shear stress $\left(\tau_{\text {os }}\right)$ was determined as the magnitude of the wall shear stress waveform as depicted.

\section{Statistical analysis.}

Statistical analysis of data within and between groups was performed with multiple ANOVA for repeated measures, followed by Student-Newman-Keuls test. A $P$ value $<0.05$ was considered to be statistically significant. All data are expressed as means $\pm \mathrm{SE}$.

\section{RESULTS}

Fourteen dogs were instrumented to obtain twelve successful experiments. Two dogs were excluded from the control group because of technical problems with instrumentation. ROS measurements for one dog in the tempol group were excluded because of inadequate dihydroethidium staining.

The hemodynamic effects of tempol and hyperglycemia are summarized in Table 1. No differences in baseline hemodynamics were observed between vehicle- and tempol-pretreated dogs. There was a small but significant increase in blood glucose concentration before the administration of $d$-glucose in tempol-treated dogs. Hyperglycemia significantly $(P<0.05)$ increased $\mathrm{LV} \mathrm{dP} / \mathrm{d} t_{\max }$ in dogs receiving vehicle, but heart rate, $\mathrm{LV}$ and aortic pressures, coronary blood flow, and LAD diameter were unchanged. In contrast, heart rate and mean arterial and LV systolic pressures were decreased during hyperglycemia in dogs pretreated with tempol. 
Hyperglycemia attenuated increases in coronary blood flow in response to acetylcholine in dogs receiving vehicle (e.g., $242 \pm 30 \%$ at baseline compared with $190 \pm 27 \%$ increase from control during moderate hyperglycemia; Fig.3). Pretreatment with tempol completely abolished this hyperglycemia-induced attenuation of coronary vasodilation in response to acetylcholine. Infusions ofd-glucose produced dose-related increases in fluorescence intensity in dogs receiving vehicle (Fig.4). In contrast, no differences in fluorescence intensity were observed during hyperglycemia in dogs pretreated with tempol.

Table 1. Systemic and coronary hemodynamics

\begin{tabular}{|c|c|c|c|}
\hline & Baseline & $\begin{array}{l}\text { Moderate } \\
\text { Hyperglycemia }\end{array}$ & $\begin{array}{l}\text { Severe } \\
\text { Hyperglycemia }\end{array}$ \\
\hline \multicolumn{4}{|c|}{$\mathrm{HR}$, beats/min } \\
\hline Vehicle & $126 \pm 7$ & $120 \pm 9$ & $129 \pm 11$ \\
\hline Tempol & $117 \pm 4$ & $99 \pm 5$ & $103 \pm 3$ \\
\hline \multicolumn{4}{|c|}{$\mathrm{MBP}, \mathrm{mmHg}$} \\
\hline Vehicle & $100 \pm 5$ & $105 \pm 5$ & $107 \pm 4$ \\
\hline Tempol & $92 \pm 4$ & $80 \pm 4, \pm$ & $84 \pm 2 *, \pm$ \\
\hline \multicolumn{4}{|c|}{ LVSP, $\mathrm{mmHg}$} \\
\hline Vehicle & $115 \pm 5$ & $118 \pm 5$ & $122 \pm 5$ \\
\hline Tempol & $103 \pm 4$ & $91 \pm 3 *, \pm$ & $95 \pm 2 *, \pm$ \\
\hline \multicolumn{4}{|c|}{ LVEDP, $\mathrm{mmHg}$} \\
\hline Vehicle & $8 \pm 1$ & $9 \pm 1$ & $8 \pm 1$ \\
\hline Tempol & $8 \pm 1$ & $8 \pm 2$ & $8 \pm 2$ \\
\hline \multicolumn{4}{|c|}{$\mathrm{dP} / \mathrm{d} t_{\max }, \mathrm{mmHg} / \mathrm{s}$} \\
\hline Vehicle & $1,640 \pm 100$ & $1,690 \pm 110$ & $1,910 \pm 170:$ \\
\hline Tempol & $1,530 \pm 130$ & $1,590 \pm 130$ & $1,780 \pm 190$ \\
\hline \multicolumn{4}{|c|}{$\mathrm{CBF}, \mathrm{ml} / \mathrm{min}$} \\
\hline Vehicle & $40 \pm 3$ & $36 \pm 4$ & $41 \pm 6$ \\
\hline Tempol & $37 \pm 4$ & $38 \pm 3$ & $40 \pm 5$ \\
\hline \multicolumn{4}{|c|}{ LAD diameter, $\mathrm{mm}$} \\
\hline Vehicle & $2.5 \pm 0.2$ & $2.6 \pm 0.2$ & $2.6 \pm 0.2$ \\
\hline Tempol & $2.1 \pm 0.1$ & $2.1 \pm 0.1 \pm$ & $2.1 \pm 0.1$ \\
\hline \multicolumn{4}{|c|}{$\begin{array}{l}\text { Blood glucose } \\
\text { concentration, } \mathrm{mg} / \mathrm{dl}\end{array}$} \\
\hline Vehicle & $69 \pm 4$ & $346 \pm 22$ & $581 \pm 13$ \\
\hline Tempol & $89 \pm 5^{ \pm}$ & $355 \pm 31 *$ & $573 \pm 6$ \\
\hline
\end{tabular}

Data are means \pm SE. Vehicle, $0.9 \%$ saline; tempol, 4-hydroxy-2,2,6,6-tetrametylpiperidine 1-oxyl; HR, heart rate; MBP, mean arterial blood pressure; LVSP and LVEDP, left ventricular (LV) systolic and end-diastolic pressures, respectively; $\mathrm{dP} / \mathrm{dtmax}$, maximal rate of increase of $\mathrm{LV}$ pressure; $\mathrm{CBF}$, coronary blood flow; $L A D$, left anterior descending coronary artery.

*Significantly $(P<0.05)$ different from baseline;

tsignificantly $(P<0.05)$ different from the respective value in the vehicle group. 


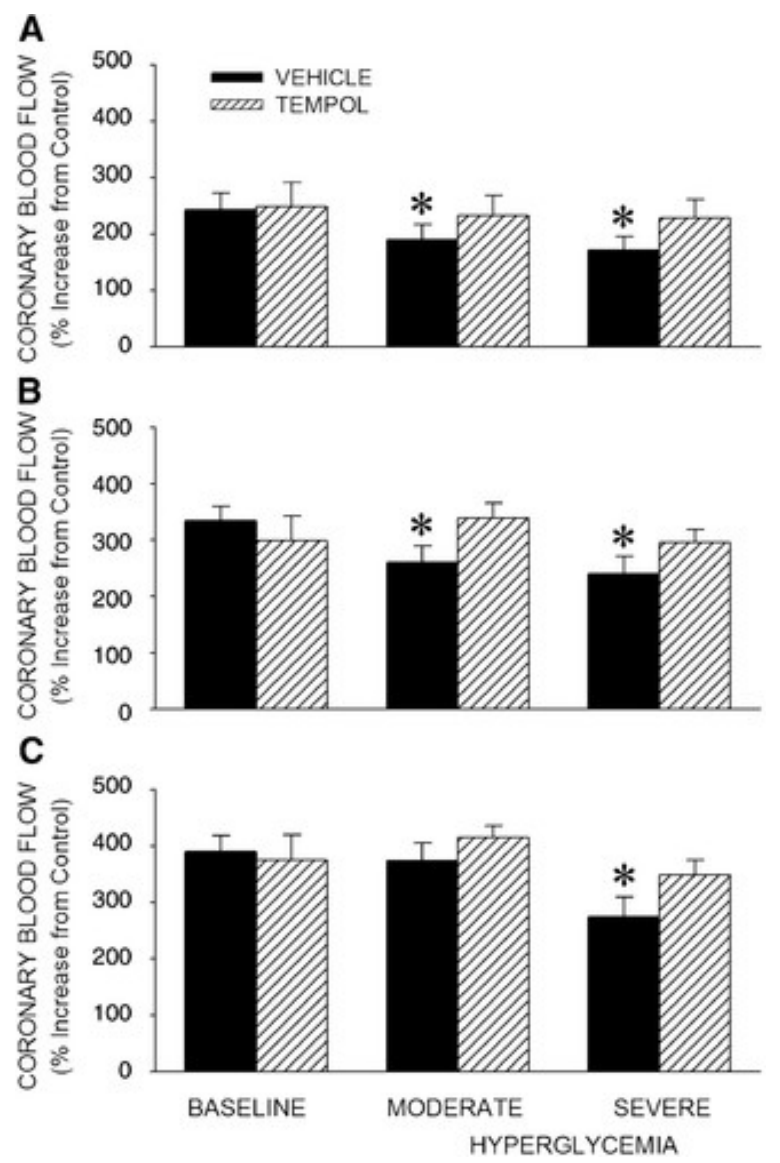

Fig. 3. Percent increase in LAD blood flow in response to left atrial administration of $10(A), 30(B)$, and $100(C)$ $\mathrm{ng} / \mathrm{kg}$ acetylcholine under control conditions and during hyperglycemia in dogs receiving vehicle and tempol. * Significantly $(P<0.05)$ different from baseline.
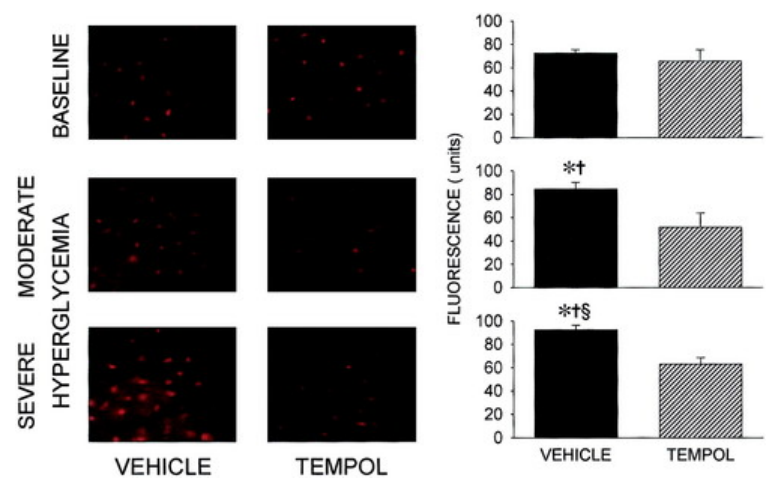

Fig. 4. Representative photomicrographs illustrate the ethidium-DNA fluorescence in nuclei of cardiac myocytes as an index of production of reactive oxygen species under control conditions and during hyperglycemia in vehicle- and tempol-pretreated dogs. Histograms depict fluorescence intensity before and during hyperglycemia in dogs receiving vehicle and tempol. * Significantly $(P<0.05)$ different from baseline; $\dagger$ significantly $(P<0.05)$ different from the respective value in tempol-pretreated dogs; §significantly $(P<0.05)$ different from moderate hyperglycemia.

No differences in blood viscosity were observed during administration of d-glucose at each shear rate in dogs pretreated with vehicle or tempol (Table 2). Pretreatment with tempol decreased blood viscosity compared with that in dogs receiving vehicle at shear rates $\leq 75 \mathrm{~s}^{-1}$ under baseline conditions. Blood viscosity was also consistently lower in dogs receiving tempol compared with vehicle during hyperglycemia. 
Table 2. Blood viscosity

\begin{tabular}{|c|c|c|c|}
\hline Shear Rate & Baseline & Moderate Hyperglycemia & Severe Hyperglycemia \\
\hline $375 \mathrm{~s}^{-1}$ & & & \\
\hline Vehicle & $3.47 \pm 0.28$ & $3.45 \pm 0.15$ & $3.42 \pm 0.08$ \\
\hline Tempol & $3.01 \pm 0.11$ & $2.91 \pm 0.13^{ \pm}$ & $2.88 \pm 0.14 \pm$ \\
\hline $187.5 \mathrm{~s}^{-1}$ & & & \\
\hline Vehicle & $3.86 \pm 0.26$ & $3.81 \pm 0.16$ & $3.87 \pm 0.08$ \\
\hline Tempol & $3.23 \pm 0.11$ & $3.16 \pm 0.15^{ \pm}$ & $3.11 \pm 0.16^{ \pm}$ \\
\hline $75 \mathrm{~s}^{-1}$ & & & \\
\hline Vehicle & $4.45 \pm 0.28$ & $4.40 \pm 0.19$ & $4.32 \pm 0.10$ \\
\hline Tempol & $3.60 \pm 0.11 \pm$ & $3.37 \pm 0.14 \pm$ & $3.40 \pm 0.17 \pm$ \\
\hline $56.25 \mathrm{~s}^{-1}$ & & & \\
\hline Vehicle & $4.85 \pm 0.35$ & $4.77 \pm 0.24$ & $4.60 \pm 0.15$ \\
\hline Tempol & $3.83 \pm 0.12 \pm$ & $3.65 \pm 0.14 \pm$ & $3.57 \pm 0.19 \pm, \pm$ \\
\hline $30 \mathrm{~s}^{-1}$ & & & \\
\hline Vehicle $^{ \pm}$ & $5.71 \pm 0.40$ & $5.53 \pm 0.31$ & $5.14 \pm 0.15$ \\
\hline Tempol & $4.36 \pm 0.17 \pm$ & $4.26 \pm 0.21 \pm$ & $4.11 \pm 0.16 \pm$ \\
\hline
\end{tabular}

Data are means $\pm \mathrm{SE}$. Vehicle, $0.9 \%$ saline.

*Significantly $(P<0.05)$ different from baseline;

tsignificantly $(P<0.05)$ different from the respective value in the vehicle group.

Global and myocardial wall shear stress were similar between vehicle- and tempol-pretreated groups under baseline conditions and during hyperglycemia (Table 3). Pericardial wall shear stress was significantly reduced in dogs pretreated with tempol compared with those receiving vehicle during severe hyperglycemia.

Hyperglycemia decreased global and myocardial oscillatory shear stress in dogs receiving vehicle but not tempol (Fig.5). In contrast, oscillatory wall shear stress at the pericardial wall was unchanged during hyperglycemia. Hyperglycemia reduced the rate of global oscillatory shear stress in dogs receiving vehicle but not tempol (Fig.6). Reductions in the rate of shear stress oscillation were also observed at the myocardial luminal surface during moderate and severe hyperglycemia in dogs pretreated with vehicle and during severe hyperglycemia in dogs pretreated with tempol. No differences were observed in the rate of oscillatory wall shear stress at the pericardial luminal surface within and between groups.

Table 3. Global and local wall shear stress

\begin{tabular}{|l|c|c|c|}
\hline & Baseline & Moderate Hyperglycemia & Severe Hyperglycemia \\
\hline Global shear stress & & & \\
\hline Vehicle & $9.4 \pm 1.5$ & $8.2 \pm 1.4$ & $8.6 \pm 1.5$ \\
\hline Tempol & $11.3 \pm 2.6$ & $8.4 \pm 1.5$ & $9.7 \pm 1.2$ \\
\hline Myocardial wall shear stress & & & $7.4 \pm 1.4$ \\
\hline Vehicle & $8.2 \pm 1.2$ & $6.8 \pm 0.6$ & $6.7 \pm 1.1$ \\
\hline Tempol & $6.6 \pm 1.5$ & $8.3 \pm 1.4$ & $11.2 \pm 1.0$ \\
\hline Pericardial wall shear stress & & & $6.8 \pm 0.8 .150$ \\
\hline Vehicle & $8.3 \pm 1.7$ & $9.7 \pm 1.2$ & \\
\hline Tempol & $7.3 \pm 0.4$ & $8.3 \pm 0.6$ & \\
\hline
\end{tabular}

Data (in dyn/cm2) are means \pm SE. Vehicle, $0.9 \%$ saline.

F3-150Significantly $(\mathrm{P}<0.05)$ different from the respective value in the vehicle group. 


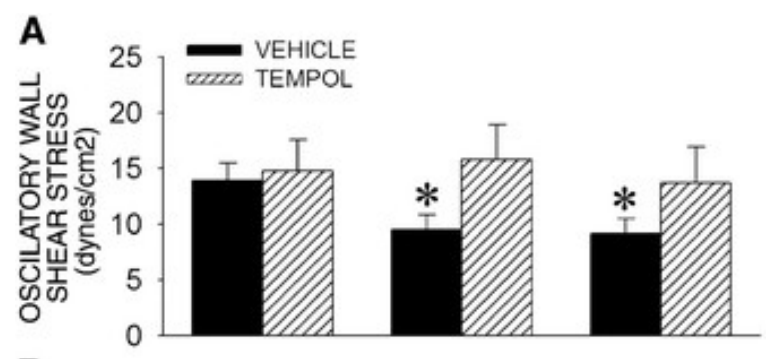

B

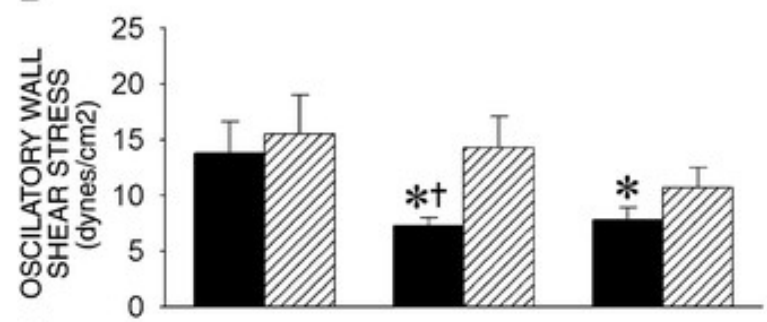

C

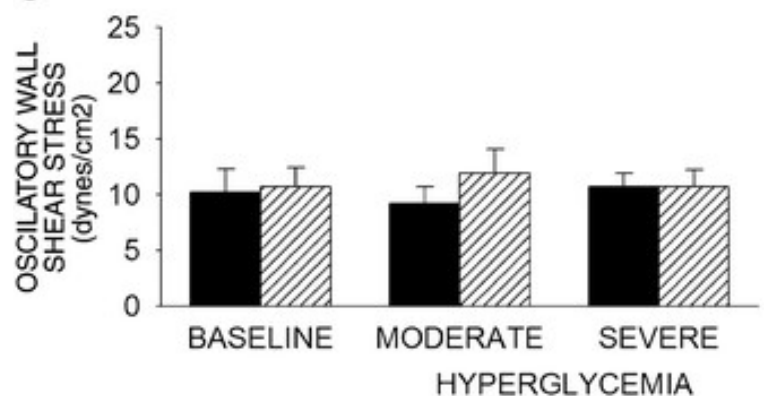

Fig. 5.Alterations in global $(A)$ and local wall shear stress at the myocardial $(B)$ and pericardial $(C)$ luminal surfaces at baseline and during hyperglycemia and in the absence (vehicle) or presence of tempol. * Significantly $(P<0.05)$ different from baseline; †significantly $(P<0.05)$ different from the respective value in dogs pretreated with tempol. 


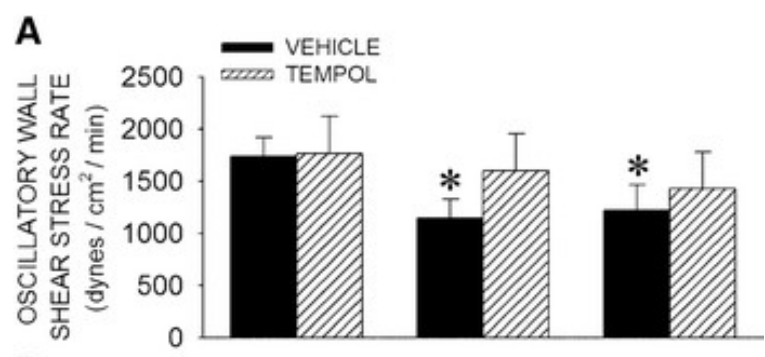

B

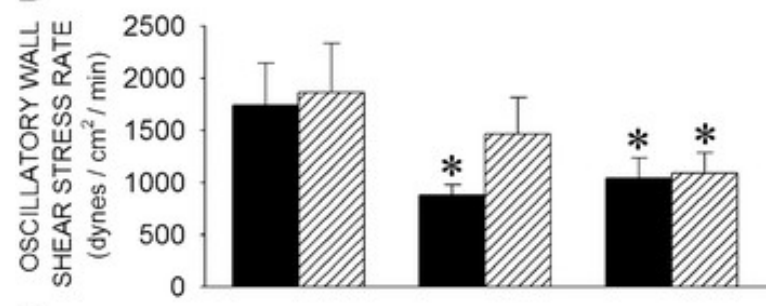

C

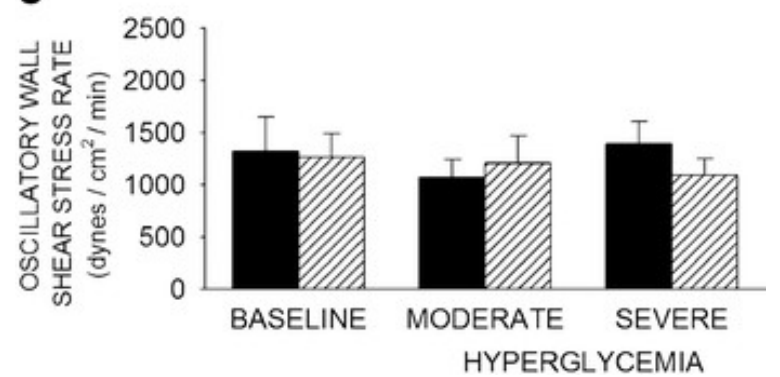

Fig. 6. Alterations in the rate of oscillatory global $(A)$, myocardial $(B)$, and pericardial $(C)$ wall shear stress under baseline conditions and during hyperglycemia in the absence (vehicle) or presence of tempol. * Significantly $(P<$ 0.05) different from baseline.

\section{DISCUSSION}

Endothelial dysfunction and increased production of ROS have been implicated in the pathogenesis of cardiovascular disease in patients with diabetes mellitus. Previous investigations demonstrated that the coronary endothelial dysfunction that occurs during hyperglycemia may be partially reversed by scavengers of oxygen-derived reactive intermediates $(2,21,27,30)$. The current results support these previous findings and further suggest that production of ROS during hyperglycemia contributes to reductions in oscillatory global and local wall shear stress in vivo. The results also demonstrate that the free radical scavenger tempol reverses hyperglycemic-induced decreases in oscillatory wall shear stress, indicating that ROS are responsible for these abnormalities in coronary fluid dynamics. The findings indicate that oscillatory but not mean wall shear stress was reduced during acute hyperglycemia. In contrast, decreases in mean wall shear stress were observed previously in arterial vessels obtained from patients with or experimental models of diabetes $(15,23)$. Whether these differences in indexes of wall shear stress observed during acute compared with chronic hyperglycemia represent a temporal adaptation to prolonged ROS generation remains to be evaluated.

Oscillatory shear stresses measured at the myocardial and pericardial luminal surfaces of the coronary artery were differentially altered by hyperglycemia in the current investigation (Fig. 5). Previous fluid dynamic studies in the LAD and left main coronary artery showed that blood flow velocity profiles are skewed toward the pericardial surface, causing the velocity profile at the myocardial surface to become less steep and resulting in lower wall shear stress at the myocardial surface compared with the pericardial surface (Fig. 1B; Refs. 9, 23). A 
greater degree of flow reversal also occurs at the myocardial compared with the pericardial surface (22). This effect preferentially establishes lower oscillatory wall shear stress with a greater deviation of the principal wall shear stress vector from the dominant flow direction at the myocardial wall. These differences in wall shear stress profiles between myocardial and pericardial surfaces have been postulated to be responsible for the preferential location of atherosclerotic plaque formation at the myocardial wall in arteries obtained from patients with coronary or vascular occlusive disease $(8,9,23)$. In contrast to low oscillatory shear stress states, increases in mean shear stress may have antiatherogenic effects by increasing superoxide dismutase (7) and NO synthase expression (40) and by increasing production of vasodilator prostanoids (33). Localized decreases in oscillatory wall stress at the myocardial wall observed during acute hyperglycemia in the current investigation may contribute to accelerated atherosclerosis during chronic hyperglycemia. This contention is supported by recent findings that endothelial dysfunction and increased vascular oxidative stress predict coronary artery disease in humans $(10,25)$. ROS production and subsequent changes in oscillatory shear stress may be a link between diabetes-induced hyperglycemia and progression of vascular disease. This intriguing hypothesis will require further investigation to confirm, however.

Hyperglycemia is well known to produce endothelial dysfunction in coronary arteries dependent or independent of diabetes $(20,27)$. The ability of tempol to restore endothelial function during hyperglycemia observed in the current investigation supports the results of a previous study in which a superoxide dismutase mimetic enhanced the vasodilator responsiveness of isolated aortic rings harvested from diabetic rats (30). Thus the current and previous studies strongly implicate a role for ROS in endothelial dysfunction during acute and chronic hyperglycemia. The present results further imply that hyperglycemia-induced endothelial dysfunction may precipitate flow conditions implicated in atherogenesis. Tempol reversed both the endothelial dysfunction (Fig. 3) and the decreases in oscillatory wall shear stress (Fig. 5) observed during hyperglycemia. These results suggest that indexes of wall shear stress may be useful as indirect indicators of oxidative stress in regions of arterial vasculature that may be susceptible to subsequent atherosclerosis.

The results of the current investigation should be interpreted within the constraints of several potential limitations. Heart rate and mean arterial pressure were lower in dogs treated with tempol than in those receiving saline during hyperglycemia. These hemodynamic effects have been previously reported with tempol (39). The decreases in heart rate may account for the reduction in the rate of oscillatory shear stress observed during the target blood glucose concentration of $600 \mathrm{mg} / \mathrm{dl}$ in tempol-pretreated dogs. However, the rates of global and myocardial surface oscillatory shear stress were lower in dogs receiving vehicle than in those pretreated with tempol during hyperglycemia (Fig. 6). These data suggest that differences in the frequency of wall shear stress oscillations exerted on the endothelium between groups were not solely attributed to differences in heart rate.

Blood viscosity was significantly reduced during the $600 \mathrm{mg} / \mathrm{dl}$ blood glucose concentration at a shear rate of 56 $\mathrm{s}^{-1}$ in dogs pretreated with tempol. This reduction in blood viscosity is probably not physiologically relevant because the minimum shear rate determined from average regional blood flow velocity and internal LAD diameter for this experimental group was $67 \mathrm{~s}^{-1}$ (data not shown). Nevertheless, equations describing the relationship of blood viscosity and shear rate below $67 \mathrm{~s}^{-1}$ were required to evaluate the dynamic behavior of blood viscosity and shear stress for temporally varying shear rates. Blood viscosities at several shear rates were also significantly lower in the tempol-compared with vehicle-pretreated dogs. However, reductions in global and myocardial oscillatory wall shear stress during hyperglycemia in dogs pretreated with vehicle were greater than those observed in tempol-treated dogs. Thus alterations in wall shear stress were probably dependent on shear rate and not blood viscosity. These results also strongly suggest that the wall shear stress data presented here may actually underestimate changes produced by hyperglycemia. 
Intravenous administration of d-glucose may have caused hyperinsulinemia in the normal dogs used for the present investigation. However, we previously demonstrated (18) that many of the effects of hyperglycemia on ischemic myocardium occur independently of plasma insulin concentration. Coronary microvascular responses to graded coronary artery occlusion are similar in hyperglycemic (hyperinsulinemic) and diabetic (insulinopenic) dogs (17). I-Arginine restores coronary collateral blood flow during hyperglycemia without altering plasma insulin concentrations (19). Thus our previous findings suggest that hyperinsulinemia is unlikely to account for the deleterious effects of hyperglycemia observed in the current or previous investigations. The presence of hyperinsulinemia may actually underestimate the impact of hyperglycemia on the coronary circulation because recent evidence indicates that insulin increases myocardial blood flow in normoglycemic patients (14).

The estimation of localized wall shear stress in arterial vessels with pulsed Doppler techniques may be prone to error for a Reynolds number typical of the blood flow through the coronary arteries (26). These errors in measurement may be attributed to the method of acquiring near-wall fluid velocity. The most precise technique assumes a fixed point on the vessel wall and then uses measured velocity points to calculate wall shear stress by least-squares interpolation. This method was implemented in the current investigation. In addition, the identical pulsed Doppler probe with a fixed insonation angle was used in each dog to minimize error. Local wall shear stress values reported previously for the LAD bifurcation (range of 5 to $33 \mathrm{dyn} / \mathrm{cm}^{2}$; Ref. 37) were also very similar to those obtained for myocardial and pericardial luminal surfaces of the LAD under control conditions in the current investigation (range of 5 to $29 \mathrm{dyn} / \mathrm{cm}^{2}$ ).

Measurement of ROS was conducted in the cardiac myocyte and not the endothelium. Recent evidence suggests that an important source of hyperglycemia-induced ROS is the mitochondrial electron transport chain within the cardiac myocyte (32). Thus determination of fluorescence intensities in myocardial nuclei identifies a likely source of ROS production that may subsequently affect coronary endothelial function. However, additional studies will be required to verify this hypothesis. Production of ROS may actually be underestimated by the dihydroethidium technique during hyperglycemia because the amount of dihydroethidium oxidized to ethidium may decrease as the rate of ROS generation increases (3). Such a situation could have occurred during severe hyperglycemia in the current investigation. Nevertheless, although measurements of ROS production may have underestimated the amount of ROS generated during hyperglycemia, the dose of tempol used in the present investigation was probably adequate to scavenge these reactive intermediates because nuclei fluorescence and endothelial function were unchanged after administration of intravenous hyperglycemia in dogs pretreated with tempol. As such, fluorescent intensity and endothelial function were unaffected by hyperglycemia for dogs in the tempol-treated group.

In summary, the present results indicate that acute hyperglycemia increases ROS production in vivo. These reactive intermediates cause endothelial dysfunction and reduce both global and local oscillatory wall shear stress in intact coronary arteries. Whether the influence of acute hyperglycemia on ROS-dependent decreases in wall shear stress and endothelial function is linked to a subsequent increase in cardiovascular risk during diabetes remains to be established.

The authors thank David A. Schwabe, John P. Tessmer, and John G. Krolikowski for technical assistance and Mary Lorence-Hanke (Department of Anesthesiology, Medical College of Wisconsin) for assistance in preparation of the manuscript.

\section{FOOTNOTES}

- $\quad{ }^{*}$ E. R. Gross and J. F. LaDisa, Jr. contributed equally to this work.

- This work was supported in part by National Institutes of Health Grants HL-03690 (to J. R. Kersten), HL63705 (to J. R. Kersten), HL-54820 (to D. C. Warltier), and GM-08377 (to D. C. Warltier). 
- Address for reprint requests and other correspondence: J. R. Kersten, Med. Coll. of Wisconsin, MEBM4280, 8701 Watertown Plank Rd., Milwaukee, WI 53226 (E-mail:jkersten@mcw.edu).

- The costs of publication of this article were defrayed in part by the payment of page charges. The article must therefore be hereby marked "advertisement" in accordance with 18 U.S.C. Section 1734 solely to indicate this fact.

- $\quad$ First published January 23, 2003;10.1152/ajpheart.01013.2002

\section{REFERENCES}

1 Altobelli SA, Nerem RM.An experimental study of coronary artery fluid mechanics.J Biomech Eng10719851623

2 Beckman JA, Goldfine AB, Gordon MB, Creager MA.Ascorbate restores endothelium-dependent vasodilation impaired by acute hyperglycemia in humans.Circulation 103200116181623

3 Benov L, Sztejnberg L, Fridovich I.Critical evaluation of the use of hydroethidine as a measure of superoxide anion radical.Free Radic Biol Med251998826831

4 Carter WO, Narayanan PK, Robinson JP.Intracellular hydrogen peroxide and superoxide anion detection in endothelial cells.J Leukoc Biol551994253258

5 Ceriello A, Quagliaro L, D'Amico M, Di Filippo C, Marfella R, Nappo F, Berrino L, Rossi F, Giugliano D.Acute hyperglycemia induces nitrotyrosine formation and apoptosis in perfused heart from rat.Diabetes51200210761082

6 Cosentino F, Hishikawa K, Katusic ZS, Luscher TF.High glucose increases nitric oxide synthase expression and superoxide anion generation in human aortic endothelial cells.Circulation9619972528

7 De Keulenaer GW, Chappell DC, Ishizaka N, Nerem RM, Alexander RW, Griendling KK.Oscillatory and steady laminar shear stress differentially affect human endothelial redox state: role of a superoxide-producing NADH oxidase.Circ Res82199810941101

8 Grottum P, Svindland A, Walloe L.Localization of atherosclerotic lesions in the bifurcation of the main left coronary artery.Atherosclerosis 4719835562

9 He X, Ku DN.Pulsatile flow in the human left coronary artery bifurcation: average conditions.J Biomech Eng11819967482

10 Heitzer T, Schlinzig T, Krohn K, Meinertz T, Munzel T.Endothelial dysfunction, oxidative stress, and risk of cardiovascular events in patients with coronary artery disease.Circulation104200126732678

11 Hillsley MV, Tarbell JM.Oscillatory shear alters endothelial hydraulic conductivity and nitric oxide levels.Biochem Biophys Res Commun293200214661471

12 Hutcheson IR, Griffith TM.Release of endothelium-derived relaxing factor is modulated both by frequency and amplitude of pulsatile flow.Am J Physiol Heart Circ Physiol2611991H257H262

13 Ilegbusi OJ, Hu Z, Nesto R, Waxman S, Cyganski D, Kilian J, Stone PH, Feldman CL.Determination of blood flow and endothelial shear stress in the human coronary artery in vivo.J Invasive Cardiol111999667674

14 lozzo P, Chareonthaitawee P, Di Terlizzi M, Betteridge DJ, Ferrannini E, Camici PG.Regional myocardial blood flow and glucose utilization during fasting and physiological hyperinsulinemia in humans.Am J Physiol Endocrinol Metab2822002E1163E1171

15 Irace C, Carallo C, Crescenzo A, Motti C, De Franceschi MS, Mattioli PL, Gnasso A.NIDDM is associated with lower wall shear stress of the common carotid artery.Diabetes 481999193197

16 Jiang Y, Kohara K, Hiwada K.Association between risk factors for atherosclerosis and mechanical forces in carotid artery.Stroke31200023192324

17 Kersten JR, Brooks LA, Dellsperger KC.Impaired microvascular response to graded coronary occlusion in diabetic and hyperglycemic dogs.Am J Physiol Heart Circ Physiol2681995H1667H1674

18 Kersten JR, Toller WG, Gross ER, Pagel PS, Warltier DC.Diabetes abolishes ischemic preconditioning: role of glucose, insulin, and osmolality.Am J Physiol Heart Circ Physiol2782000H1218H1224

19 Kersten JR, Toller WG, Tessmer JP, Pagel PS, Warltier DC. Hyperglycemia reduces coronary collateral blood flow through a nitric oxide-mediated mechanism.Am J Physiol Heart Circ Physiol2812001H2097H2104 
20 Kocsis E, Pacher P, Posa I, Nieszner E, Pogatsa G, Koltai MZ. Hyperglycaemia alters the endotheliumdependent relaxation of canine coronary arteries.Acta Physiol Scand1692000183187

21 Kocsis E, Posa I, Koltai MZ, Pogatsa G.Impaired endothelium-dependent vasodilation in hyperglycaemic dogs in vivo (Abstract).J Mol Cell Cardiol342002A34

22 Ku DN.Blood flow in arteries.Annu Rev Fluid Mech291997399434

$23 \mathrm{Ku} \mathrm{DN}$, Giddens DP, Zarins CK, Glagov S.Pulsatile flow and atherosclerosis in the human carotid bifurcation. Positive correlation between plaque location and low oscillating shear stress.Arteriosclerosis51985293302

24 LaDisa JF, Hettrick DA, Olson LE, Guler I, Gross ER, Kress TT, Kersten JR, Warltier DC, Pagel PS.Stent implantation alters coronary artery hemodynamics and wall shear stress during maximal vasodilation.J Appl Physiol93200219391946

25 Landmesser U, Harrison DG.Oxidant stress as a marker for cardiovascular events: Ox marks the spot.Circulation104200126382640

26 Markou CP, Ku DN.Accuracy of velocity and shear rate measurements using pulsed Doppler ultrasound: a comparison of signal analysis techniques.Ultrasound Med Biol171991803814

27 Matsunaga T, Okumura K, Ishizaka H, Tsunoda R, Tayama S, Tabuchi T, Yasue H.Impairment of coronary blood flow regulation by endothelium-derived nitric oxide in dogs with alloxan-induced diabetes.J Cardiovasc Pharmacol2819966067

28 Miller FJ, Gutterman DD, Rios CD, Heistad DD, Davidson BL.Superoxide production in vascular smooth muscle contributes to oxidative stress and impaired relaxation in atherosclerosis.Circ Res 82199812981305

29 Mugge A, Elwell JH, Peterson TE, Harrison DG. Release of intact endothelium-derived relaxing factor depends on endothelial superoxide dismutase activity.Am J Physiol Cell Physiol2601991C219C225

30 Nassar T, Kadery B, Lotan C, Da'as N, Kleinman Y, Haj-Yehia A.Effects of the superoxide dismutase-mimetic compound tempol on endothelial dysfunction in streptozotocin-induced diabetic rats.Eur $\mathbf{J}$ Pharmacol4362002111118

31 Nichols W, O'Rourke MF.McDonald's Blood Flow in Arteries: Theoretic, Experimental, and Clinical Principles4th ed.19980xford Univ. PressNew York

32 Nishikawa T, Edelstein D, Du XL, Yamagishi S, Matsumura T, Kaneda Y, Yorek MA, Beebe D, Oates PJ, Hammes HP, Giardino I, Brownlee M.Normalizing mitochondrial superoxide production blocks three pathways of hyperglycaemic damage.Nature4042000787790

33 Okahara K, Sun B, Kambayashi J.Upregulation of prostacyclin synthesis-related gene expression by shear stress in vascular endothelial cells.Arterioscler Thromb Vasc Biol18199819221926

34 Pieper GM.Acute amelioration of diabetic endothelial dysfunction with a derivative of the nitric oxide synthase cofactor, tetrahydrobiopterin.J Cardiovasc Pharmacol291997815

35 Rubanyi GM, Vanhoutte PM.Superoxide anions and hyperoxia inactivate endothelium-derived relaxing factor.Am J Physiol Heart Circ Physiol2501986H822H827

36 Tan KC, Chow WS, Ai VH, Metz C, Bucala R, Lam KS.Advanced glycation end products and endothelial dysfunction in type 2 diabetes.Diabetes Care25200210551059

37 Tang TD, Giddens DP, Zarins CK, Glagov S.Velocity profile and wall shear stress measurements in a model human coronary artery.Advs Bio-Eng171990261263

38 Tesfamariam B, Cohen RA.Free radicals mediate endothelial cell dysfunction caused by elevated glucose.Am J Physiol Heart Circ Physiol2631992H321H326

39 Xu H, Fink GD, Chen A, Watts S, Galligan JJ.Nitric oxide-independent effects of tempol on sympathetic nerve activity and blood pressure in normotensive rats.Am J Physiol Heart Circ Physiol2812001H975H980

40 Ziegler T, Silacci P, Harrison VJ, Hayoz D.Nitric oxide synthase expression in endothelial cells exposed to mechanical forces. Hypertension321998351355 